\title{
Database of Czech Forest Classification System
}

\author{
Václav Zouhar
}

\begin{abstract}
The Database of the Czech Forest Site Classification System (DCFCS; GIVD ID EU-CZ-002) is focused on data about ecological conditions and structure of forest ecosystems in the territory of the Czech Republic. This database is managed by Forest Management Institute Brandýs nad Labem. DCFCS consists of two parts, the numeric database and the GIS data. The numeric database includes all descriptive data of sample plots used in classification. Each plot is characterized by features of ecotope (information about geomorphology, climate, pedology etc.) and vegetation data (information on composition and structure). Maps of site types with the position of sample plots are represented as GIS data.
\end{abstract}

Keywords: Czech Republic; Forest Site Classification.

GIVD Database ID: EU-CZ-002

\section{Database of Czech Forest Classification System}

Scope: Data of forest ecosystems from the Czech Republic.

Status: completed and continuing

Period: 1926-2005

Database manager(s): Václav Zouhar (zouhar.vaclav@uhul.cz)

Owner: Forest Management Institute Brandys nad Labem

UHUL Brandys nad Labem

Nabrezni 1326

25001 Brandys nad Labem

Czech Republic

Web address: [NA]

Availability: according to a specific agreement

Database format(s): TURBOVEG

Publication: [NA]

Plot type(s): normal plots; time series

Non-overlapping plots: 32,387

Total plot observations: 48,439

Countries: CZ: $100.0 \%$

Forest: [NA] — Non-forest: [NA]

Guilds: all vascular plants: $100 \%$

Environmental data: altitude: $99 \%$; slope aspect: $82 \%$; slope inclination: $98 \%$; microrelief: $63 \%$; surface cover other than plants (open soil, litter, bare rock etc.): $99 \%$; other soil attributes: $100 \%$

Performance measure(s): cover: $100 \%$

Geographic localisation: point coordinates less precise than GPS, up to $1 \mathrm{~km}: 100 \%$

Sampling periods: 1950-1959: 26.0\%; 1960-1969: 36.0\%; 1970-1979: 28.0\%; 1980-1989: 2.0\%; 1990-1999: 5.0\%; 2000-2009: 3.0\% Information as of 2012-07-12; further details and future updates available from http://www.givd.info/ID/EU-CZ-002

Václav Zouhar (zouhar.vaclav@uhul.cz)

Forest Management Institution, Vrazova 1, 61600 Brno, CZECH REPUBLIC 